\title{
Das Projekt "Deutsch als Zweitsprache in Dialektumgebung"
}

\author{
Andrea Ender/Wei Li/Katharina Straßl (Bern)
}

\begin{abstract}
This paper presents the ongoing project "German as a Second Language in a Dialect-Speaking Environment" which is concerned with the acquisition of German as a second language by immigrant children in the German-Speaking part of Switzerland. As this is one of the areas where a distinct dialect coexists with the standard variety, the language acquisition poses a challenge for immigrant children: The daily input does not correspond at all, or only in part, to what the immigrant children should learn (e.g. for achievement in school and career opportunities), namely standard German. The aims of the project are to evaluate existing language measuring methods for preschool children and to develop a test that is adapted to the bilingualism of the immigrant children and the specific diglossic learning situation. Furthermore, an empirical pilot study deals with the language abilities of primary school children and examines how and to what extend the local dialect influences the use of standard German in spoken and written texts.
\end{abstract}

\section{$1 \quad$ Einleitung}

In der deutschsprachigen Schweiz sind wir mit einer Situation konfrontiert, die traditionell als Diglossie bezeichnet wird (cf. Ferguson 1959). Dieses Nebeneinander von verschiedenen Varietäten stellt besonders für Sprachlernende eine besondere Herausforderung dar, da der tägliche Input nicht oder nur teilweise dem entspricht, was das Ziel des Zweitspracherwerbs darstellt: die deutsche Standardsprache. Offen ist jedoch, wie und in welchem Umfang der Erwerb der Standardsprache durch die lokalen Dialekte beeinflusst wird. Obwohl diese Frage in der Schweiz immer mehr ins öffentliche Interesse gerückt wird, können in der Diskussion kaum wissenschaftliche Ergebnisse herangezogen werden. Das laufende Projekt soll hier einen wesentlichen und ausstehenden Beitrag im Bereich des Arbeitsgebietes Deutsch als Zweitsprache leisten, denn die "Diglossie als mögliche Verursacherin von Erwerbsproblemen ist nach wie vor wenig erforscht und entsprechend umstritten" (Gyger 2005: 13).

Das Projekt konzentriert sich hierbei auf zwei Bereiche: Zum einen soll ein theoretisch fundiertes und für die Praxis erprobtes Sprachstandsmessungsverfahren für Vorschulkinder erarbeitet und für die Dialektsituation adaptiert werden. Zum anderen wird anhand von gesprochenen und geschriebenen Texten von Primarschul-Migrantenkindern analysiert, ob und wie sich Einflüsse der Dialektumgebung auf den Hochdeutscherwerb - besonders auf den Ebenen Morphologie und Syntax - auswirken.

\section{Die Spracherwerbssituation der Migrantenkinder in der Schweiz}

In der Beschreibung der diglossischen Situation wurden verschiede Aspekte des Nebeneinanders von Dialekt und Standard hervorgehoben, wobei die Wichtigkeit des Kommunikationsmediums, die Relevanz der Unterscheidung zwischen Produktion und Rezeption, die Wertschätzung und Haltung der Sprachgemeinschaft und andere Aspekte diskutiert wurden (cf. Kolde 1981; Werlen 1998; Berthele 2004). Der Dialekt dient in der 
Deutschschweiz durch sämtliche soziale Klassen hindurch grundsätzlich als identitätsstiftendes Merkmal und ist Ausdruck lokaler Zugehörigkeit. Kurz zusammengefasst lässt sich die Sprachsituation folgendermaßen beschreiben: Für alltägliche Kommunikation in beinahe allen Kontexten, für viele Rundfunksendungen und immer mehr auch für informelle schriftliche Texte wie E-Mails und SMS wird der Dialekt gewählt. Die Standardsprache wird neben ihrer traditionellen Rolle als Medium für geschriebene Kommunikation in der gesprochenen Sprache beinahe ausschließlich für institutionalisierte Formen der Kommunikation (z. B. im schulischen und akademischen Kontext), für Nachrichtensendungen im Rundfunk und für die Kommunikation mit Personen verwendet, die die lokale Mundart nicht verstehen.

Besonders interessant ist die Situation im vorschulischen und schulischen Kontext. Der Standarddeutscherwerb beginnt grundsätzlich erst mit dem Schuleintritt, da im Kindergarten der Gebrauch des Dialekts dominiert (cf. Landert 2006: 211). In den vergangenen Jahren wurde jedoch auch in Kindergärten der konsequente Gebrauch des Hochdeutschen versuchsweise eingeführt ${ }^{1}$. In den ersten Schuljahren benützen die Lehrpersonen hauptsächlich die Mundart und versuchen die Kinder langsam an das Verstehen und den Gebrauch der Standardsprache hinzuführen. Allerdings kann davon ausgegangen werden, dass die meisten Kinder bereits bei der Einschulung über Fähigkeiten zumindest im rezeptiven Umgang mit der Standardsprache verfügen (cf. Häcki Buhofer/Burger 1998). Im weiteren Verlauf der Schulzeit gewinnt dann ein situativer Gebrauch des Hochdeutschen an Bedeutung (cf. Stöckling 2005; Landert 2006). Die Standardsprache wird in der Funktion einer Schriftsprache gelernt und mündlich vorwiegend in Kontexten verwendet, in denen Leistungskontrollen im Vordergrund stehen, während der lokale Dialekt in unmarkierten bzw. entspannten Kontexten zum Einsatz kommt. Es liegt auf der Hand, dass sich dieser Gebrauch negativ auf Routinen sowie auf die Einstellung gegenüber den Varietäten auswirken könnte. Daher werden gerade in den letzten Jahren - im Einklang mit kantonalen Weisungen ${ }^{2}$ immer häufiger Stimmen laut, die sich für den grundsätzlichen Einsatz der Standardsprache im schulischen Bereich aussprechen. Dies sollte sich für Schweizer Kinder, besonders jedoch auch für mehrsprachige Kinder mit Migrationshintergrund positiv auf den Spracherwerb und die Schriftaneignung auswirken.

Der Sprach- und Schrifterwerb von Migrantenkindern vollzieht sich in der Schweiz im Spannungsfeld zwischen Dialekt und Hochsprache und je nach Kontext sind die Kinder mit beiden Varietäten und vor allem mit einem Wechseln zwischen den Varietäten konfrontiert. Die Kinder lernen aufbauend auf ihrer Erstsprache das Hochdeutsche als Zweitsprache bzw. sie lernen den Dialekt als Zweitsprache und daneben die standardsprachliche Varietät. In der Diskussion um den Zweitspracherwerb von Migrantenkindern (cf. Graf 1987, Apeltauer 1997, Engin et al. 2004) wird eine solche Situation meist vernachlässigt. Nur in Ausnahmefällen wie zum Beispiel von Nodari/De Rosa (2003: 24) wird darauf hingewiesen, dass es sich bei der Sprache der Umgebung durchaus auch um einen Dialekt handeln kann, was das Zusammenspiel der verschiedenen Sprachen/Varietäten im mündlichen und schriftlichen Bereich komplexer macht.

\section{$3 \quad$ Mögliche Einflüsse der Diglossie auf den Standardspracherwerb}

Gut ausgebildete Fähigkeiten in der Standardsprache sind eine wesentliche Voraussetzung für eine erfolgreiche Bildungslaufbahn und die bestmögliche Entwicklung diese Fähigkeiten steht für Kinder daher im Vordergrund. Dass nun das Nebeneinander von Varietäten, die sich

\footnotetext{
${ }^{1}$ Von Erfahrungen aus der wissenschaftlichen Begleitung solcher Projekte berichtet Gyger (in diesem Band).

${ }^{2}$ So präzisiert beispielsweise der Berner Lehrplan seit 1. August 2007, dass Hochdeutsch ausnahmslos in allen Fächern verwendet werden soll (cf. Pulver 2007: 14f.).
} 
deutlich unterscheiden, auch Einflüsse auf den Erwerb des Standards hat, scheint intuitiv einleuchtend. Die Gemeinsamkeit aller bestehenden Spracherwerbstheorien liegt in der Annahme, dass Spracherwerb vor allem mittels Verarbeitung und Interpretation von Sprachmaterial erfolgt (cf. Mitchell/Myles 2004: 20). Da der Spracherwerb wie jedes andere Lernen von fruchtbaren Lernsituationen abhängt, nimmt der Input eine zentrale Rolle ein. Durch die vielen situativen Einschränkungen, denen das Hochdeutsche unterliegt, verringern sich für Sprachlernende in der Deutschschweiz der Input in der Standardvarietät und die potentiellen Lernsituationen.

Bislang ist jedoch unklar, inwiefern der Zweitspracherwerb der Kinder durch diese Sprachsituation beeinträchtigt wird. Von pädagogischer Seite wird beispielsweise gemutmaßt, dass das Wechseln zwischen den Varietäten bei den Kindern im Spracherwerb zu Verwirrung führt, da sie nur unzureichend die Fähigkeit besitzen, zwischen den Varietäten zu unterscheiden: "Code-Switching beeinträchtigt jedoch den Zweitspracherwerb von Kindern mit Migrationshintergrund. Selbst Kinder, die in der Schweiz geboren oder seit Jahren hier wohnhaft sind, können Mundart und Standardsprache nur schwer unterscheiden" (PHZH und EDK-Ost 2005: 4). Um diese Behauptung zu be- oder widerlegen, fehlen bisher jedoch wissenschaftliche Ergebnisse.

In struktureller Hinsicht können syntaktische und morphologische Merkmale, die Standardsprache und Mundart unterscheiden, zu Interferenzen führen. Das bereits vorhandene System der Mundart bzw. das im Input Häufigere wirkt auf das zu Lernende, nämlich die Hochsprache. Der lokale Dialekt könnte den Zweitspracherwerb somit beispielsweise in den Bereichen des Kasusgebrauchs, im Gebrauch der verbalen Tempora, im Gebrauch der klitischen und nichtklitischen Personalpronomina, bei Relativanschlüssen mit wo, eingeleitetenen Infinitivsätzen mit für zum etc. beeinflussen. ${ }^{3}$ Im Folgenden werden einige Beispiele für Interferenzen aus dem Dialekt bei deutscherwerbenden Kindern angeführt:

- $\quad$ Es war einmal eine Familie, wo sehr glücklich war.

Hier wird der Relativanschluss mit wo anstelle von die gebildet.

- $\quad$ Einmal habe ich mit meinem Kolleg das Velo getauscht.

Hier sind im Lexikon zwei varietätenbedingte Besonderheiten zu erkennen. Zum einen das Wort Velo anstelle von Rad und zum anderen der Ausdruck Kollege. Letzterer trägt im schweizerischen sowie österreichischen Hochdeutschen die Bedeutung 'Freund' (cf. Ammon 2004: 424). Der Fehler liegt hier jedoch in der fehlenden morphologischen Markierung, denn das Substantiv ist nicht vollständig dekliniert, es fehlen die Auslaute.

- Der Herr Doktor Hoessly sagte mir, ich müsse ins Bett liege.

Die Phrase ins Bett liege ist stark vom Dialekt geprägt und weicht in mehreren Bereichen von der Standardnorm ab. Zum einen in der Wertigkeit des Verbs, zum anderen in der Realisierung des Infinitivs. Richtig müsste es heißen: Ich müsse mich ins Bett legen. Der Fehler lässt sich durch die unterschiedliche Wertigkeit bzw. Bedeutung von liegen im Dialekt erklären. Dort kann es durchaus für einen Vorgang und mit einem Präpositionalobjekt im Akkusativ verwendet werden. Außerdem wird die Verbform liegen falsch realisiert. Denn beim Infinitiv liegen fehlt das auslautende $n$ - ebenso wie dies in der Dialektaussprache des Infinitivs der Fall ist.

Solche Beispiele aus Texten von Migrantenkindern können als Indiz dafür betrachtet werden, dass die Dialektumgebung den Standardspracherwerb beeinflusst. Ein solcher Einfluss wurde

\footnotetext{
${ }^{3}$ Das SNF-Projekt „Dialektsyntax des Schweizerdeutschen“ beschäftigt sich am Deutschen Seminar Zürich unter der Leitung von Elvira Glaser mit der sprachgeographischen Gliederung der Deutschschweiz und verfolgt dabei dass Ziel eines syntaktischen Atlas des Schweizerdeutschen zu erstellen (www.ds.uzh.ch/dialektsyntax/).
} 
bisher erst vereinzelt konkret nachgewiesen, so z. B. in einer Untersuchung von Heide Wegener (1994) zur Nebensatzbildung von Migrantenkindern im süddeutschen Raum. Bei ihrer Testgruppe zeigte sich in der Nebensatzbildung mit weil, in Possessivkonstruktionen und bei der Kasuszuweisung in Präpositionalphrasen ein deutlicher und langfristiger Einfluss des Umgebungsdialekts. In einer fehlerlinguistischen Untersuchung von Schülertexten in Berlin deckt auch Rosenberg (1986) Einflüsse des Dialekts auf den Erwerb des Standards sowohl auf phonologischer wie auch auf morphosyntaktischer Ebene auf.

Für die Schweiz fehlen bislang detaillierte quantitative und qualitative Analysen. Inwiefern der Dialekt bei diesem Lern- und Entwicklungsprozess eine Wirkung zeigt und wie diese erhoben und festgemacht werden kann, steht bei der Untersuchung zweier unterschiedlicher Altersstufen im Mittelpunkt unseres Forschungsprojekts.

\section{Ein Sprachstandsmessungsverfahren für das Vorschulalter in der diglossischen Situation}

\subsection{Die Ausgangslage}

Wie eingangs erwähnt, ist es eines der Ziele des Projekts, ein aussagekräftiges Testinstrument zur Feststellung des Hochsprachstands von Kindern mit Migrationshintergrund im Vorschulalter in der Deutschschweiz zu entwickeln, um spezifische Schwierigkeiten beim Hochspracherwerb in der diglossischen Sprachsituation zu erkennen.

In den letzten Jahren wurden sehr viele Sprachstandsmessungen entwickelt. Auf dem deutschsprachigen Testmarkt ist ein regelrechter Boom der Sprachstandsinstrumentsentwicklung zu beobachten (cf. Fried 2004, Ehlich 2005). Sprachstandserhebungen können pädagogisch (Sprachförderung), bildungspolitisch (Einschulung) oder medizinisch (Therapie) motiviert sein, bei ihnen allen stellen sich aber dieselben grundlegenden Fragen: Was wird gemessen bzw. was ist das Ziel der Sprachstandserhebung? Wie wird gemessen? Wie wird ausgewertet oder wie werden die ermittelten Messwerte beurteilt?

$\mathrm{Zu}$ den am häufigsten eingesetzten Testverfahren im deutschsprachigen Raum gehören Bärenstark (2002), CITO (2007), Deutsch Plus (2006), Fit in Deutsch (2006), HAVAS 5 (2003), Kenntnisse in Deutsch als Zweitsprache (2002), MSS (2003), SFD (2006), SISMIK (2006) und SSV (2003). Die Erhebungsmethoden, die in diesen Tests zum Einsatz kommen, sind teilnehmende Beobachtung, Interviews, freie Gespräche, Nacherzählungen oder das Erzählen von Geschichten sowie auch Einzeltests zu phonetisch-phonologischen, morphologischen, lexikalischen oder syntaktischen Kompetenzen. Einige dieser Tests berücksichtigen zwar mögliche Einflüsse aus der L1, keiner jedoch Einflüsse aus dem Umgebungsdialekt. Die üblichen Tests sind deshalb nur bedingt dafür geeignet, den Sprachstand von Kindern mit Migrationshintergrund in der Schweiz zu messen, denn die Schwierigkeiten, die die überwiegende Mehrzahl der Kinder mit nicht-deutscher Muttersprache in der Deutschschweiz beim L2-Erwerb haben, unterscheiden sich sowohl von den Schwierigkeiten sprachauffälliger als auch von jenen kognitiv benachteiligter einsprachiger Kinder. Kinder nicht-deutscher Muttersprache haben häufig Probleme beim Entdecken von regelgeleiteten Strukturen (cf. Penner 2005: 61f.), die bei Kindern im Vorschulalter noch in der Form vorbewusster, impliziter Kenntnisse über eine Sprache verharren. Deshalb sind sowohl in der Sprachrezeption als auch insbesondere in der Sprachproduktion Probleme zu erwarten, so etwa Unsicherheiten bei der Genuszuweisung oder bei der Pluralbildung. Die Unterschiede zwischen Mundart und Hochdeutsch könnten die Regelableitung zusätzlich erschweren, weshalb solche Unterschiede im geplanten Testverfahren explizit berücksichtigt werden. 


\subsection{Der Aufbau des Testverfahrens}

Das geplante Testverfahren orientiert sich an folgenden zentralen Leitlinien:

- Anpassung an den kindlichen Sprachstand: Die Testinhalte müssen dem altersspezifischen kognitiven Entwicklungsstand der Kinder entsprechen.

- Standardsprachkompetenz: Gegenstand des Tests ist die standardsprachliche Kompetenz der Migrantenkinder mit Berücksichtigung möglicher Einflüsse des Dialekts.

- Zielgruppe: der Test ist ausschließlich für Migrantenkinder mit nichtdeutscher Erstsprache konzipiert.

Um ein möglichst umfassendes Bild der sprachlichen Kompetenzen der Kinder zu erhalten, umfasst das Testverfahren unterschiedliche Erhebungsmethoden, nämlich (1) Beobachtung der Kinder im Kindergartenalltag, (2) gesteuerte Interviews mit Lehrkräften, Eltern und dem $\mathrm{zu}$ testenden Kind zur Fremd- und Selbeinschätzung des Sprachentwicklungsstands der Kinder aus verschiedenen Perspektiven sowie (3) einen Test der Sprachkompetenz im engeren Sinne.

Durch die Beobachtung im Kindergarten werden Informationen zum alläglichen Sprachgebrauch dokumentiert, wie z. B. Sprachverwendung der Kinder in expliziten Hochspracheinheiten sowie im freien Spiel, Sprachverwendung der Lehrkraft.

Nachdem zunächst mit Hilfe eines Fragebogens allgemeine Informationen über das Sprachprofil des zu testenden Kindes gesammelt wurden, werden sodann in einem gesteuerten Interview mit der Lehrkraft Daten zu Sprachverwendung, Sprachverhalten und Sprachkompetenz des Kindes erhoben, etwa: Spricht das Kind im Dialekt/auf Hochdeutsch deutlich? Beantwortet das Kind Fragen sinngemäß und verständlich im Dialekt/auf Hochdeutsch? Versteht es verbale Instruktionen auf Hochdeutsch? Stellt es Fragen auf Hochdeutsch? etc. All diese Fragen zielen auch darauf ab, Differenzen zwischen dem Erwerbsstand im Hochdeutschen und der Mundart festzustellen. Beim Interview mit den Eltern werden in ähnlicher Weise sowohl die Sprachgewohnheiten innerhalb der Familie als auch Sprachverhalten und Sprachkompetenz des Kindes erfragt. Daneben werden die Einstellungen der Eltern gegenüber den Sprachen und Sprachvarietäten (der Mundart und dem Hochdeutschen) durch Fragen zur Wichtigkeit von Hochdeutsch- bzw. Mundartkompetenz expliziert. Das Interview mit dem Kind widmet sich Fragen zur Einschätzung der eigenen Sprachkompetenz, dient jedoch vor allem auch dazu, aktive Sprachäußerungen des Kindes zu provozieren.

Der dritte Teil der Erhebung überprüft die Hochsprachkompetenz in Produktion und Rezeption, wobei besonders das Ausmaß der Beherrschung grammatischer Regeln stichprobenartig zu erfassen versucht wird. Einer oft eingesetzten Methode folgend hört sich das Kind die Tonbandaufnahme einer kurzen Geschichte an und wird dazu aufgefordert, einige zur Geschichte passende Bilder in die richtige Reihenfolge zu bringen, $w$-Fragen zu beantworten und die ganze Geschichte anhand der Bilder mit eigenen Worten nachzuerzählen. Einerseits werden die rezeptiven Kompetenzen im Hochdeutschen überprüft, indem das Kind die dem Kontext entsprechenden Zusammenhänge zwischen den Bildern erkennt und auf den Inhalt bezogene Fragen beantwortet. Andererseits wird die auf Hochdeutsch nacherzählte Geschichte aufgenommen und in Hinblick auf dialektbedingte Fehler untersucht.

Um dialektale Einflüsse im Bereich der Morphologie zu erheben, werden mithilfe von Bildkarten hochdeutsche Äußerungen elizitiert, in denen das Kind hochdeutsche Plurale bilden und Präpositionen verwenden muss. Da sich Pluralbildung und Präpositionengebrauch 
im Hochdeutschen und dem Umgebungsdialekt abweichen, ist zu vermuten, dass es in diesen Bereichen zu negativem Transfer kommt.

In einer Pilotstudie soll untersucht werden, ob sich dieses Verfahren für die Erhebung von Dialekteinflüssen im Standardspracherwerb bewährt. Mit der Frage, ob und wie sehr sich der Einfluss des Dialekts auch längerfristig auf die Sprachkompetenz der Kinder mit Migrationshintergrund auswirkt, beschäftigt sich die zweite Untersuchung.

\section{$5 \quad$ Eine Studie zu Dialekteinflüssen in Texten von Primarschulkindern}

\subsection{Die Ausgangslage}

Wie eingangs bereits erläutert, stehen diejenigen SchülerInnen, die Deutsch als Zweitsprache erwerben, in der Deutschschweiz aufgrund der diglossischen Sprachsituation, einer besonderen Herausforderung gegenüber. Darauf basierend beleuchtet diese Studie bei Primarschulkindern im Alter von 9-11 Jahren die Frage nach dem vermuteten Einfluss, den der lokale Dialekt nach mehreren Jahren schulischen Zweitspracherwerbs ausübt.

Das Hauptaugenmerk der Untersuchung ist aufgrund der Kürze der verfügbaren Zeit auf Morphologie und Syntax gerichtet; nicht analysiert werden unter anderem das Lexikon und die phonetisch/phonologische Ebene. Zudem wird auch auf eine Analyse der Textstruktur und -kohäsion verzichtet ${ }^{4}$. Ebenso können aus Gründen der Machbarkeit auch soziale Faktoren wie Alter, Motivation, Lernbedingungen etc. nur am Rande mitberücksichtigt werden.

\subsection{Der Aufbau der Untersuchung}

Die ProbandInnen der Untersuchung sind Primarschulkinder im Alter von 9-11 Jahren aus sieben verschiedenen Klassen. In diesen Klassen wurden schriftliche und mündliche Daten von insgesamt 61 Kindern gesammelt, wobei neben den Kindern mit Migrationshintergrund jeweils einige Deutschschweizer Kinder als Vergleichsgruppe hinzugezogen wurden. In der Untersuchungsgruppe waren insgesamt 14 Erstsprachen vertreten. Um die Analysen möglichst vergleichbar zu gestalten, können nicht alle Sprachgemeinschaften berücksichtigt werden. Da die meisten befragten Kinder mit Migrationshintergrund den beiden Sprachgruppen Bosnisch/Kroatisch/Serbisch (BKS) und Türkisch (T) angehören, bilden jeweils acht Kinder aus diesen beiden Sprachgruppen und zusätzliche acht Kinder aus der Vergleichsgruppe die Grundlage für eine detaillierte Analyse.

Der Untersuchung liegt ein Korpus von geschriebenen und gesprochenen Texten zu Grunde. Bei den vorliegenden schriftlichen Daten handelt es sich zum einen um Texte, die bereits im Regelunterricht im Zeitraum von Februar bis April 2007 entstanden sind. Da an der Untersuchung mehrere Klassen dreier Schulstufen teilgenommen haben, besteht das Korpus aus einer bunt gemischten Palette an Aufsätzen, von Erlebniserzählungen über Bildgeschichten bis hin zu Nacherzählungen, die sich in Länge und Leistungsniveau erheblich unterscheiden.

In einem zweiten Schritt konnten noch Texte gesammelt werden, die die Kinder zum selben Thema, mit derselben Themeneinführung und auch innerhalb der gleichen Zeitspanne (Dauer einer Unterrichtsstunde) verfassten, nämlich eine Reizwortgeschichte zu den Wörtern Schulschluss - Gewitter - Regenschirm. Hier ist nun ein direkter Vergleich dessen möglich, was die beteiligten Kinder in der gleichen Zeit und zum selben Thema sprachlich produzieren können und inwiefern sich die Resultate voneinander unterscheiden. Anhand dieser Texte sind direkte Vergleiche möglich.

\footnotetext{
${ }^{4}$ Empirische Untersuchungen zur Textstruktur und -kohärenz führten u. a. bereits Schmidlin (1999) und Knapp (1997) durch.
} 
Die mündlichen Daten wurden mittels Interviews erhoben. Gezielte Interview-Fragen liefern des Weiteren auch Auskünfte über das Sprachverhalten und über die Sprachgewohnheiten der Kinder: Hast du Geschwister und in welcher Sprache sprichst du mit ihnen? Wie sprichst du mit deiner Mutter/deinem Vater? Wie sprecht ihr alle miteinander? In welcher Sprache sprichst du mit deinen Freunden in der Schule/in der Freizeit? oder auch: Welche Sprache sprichst du am liebsten? Welche Sprache glaubst du, dass du am besten sprichst? Was machst du am liebsten in deiner freien Zeit? etc.

Um auch im Bereich des Mündlichen zusammenhängende Erzählungen zu erhalten, wurden die Kinder des Weiteren aufgefordert, den Inhalt ihres Lieblingsbuches und ihrer Lieblingsfernsehserie bzw. ihres Lieblingsfilms zu erzählen. Ferner wurden die Kinder gebeten, sich eine sechs Illustrationen umfassende Bildgeschichte anzusehen und diese zu erzählen. Folgendes Beispiel zeigt in transkribierter Form eine Passage besagter Bildgeschichte, erzählt von einem serbischen Jungen:

es handelt sich um einen jungen und einen wie ein sein vater, sie bauten ein, wie soll ich sagen, ein schlitten und sie fahrten auf schnee und dann war der schlitten, ja wie soll ich sagen, sie stürzten ab, der schlitten war ein bisschen gebrochen, äh wie kaputt, und dann mussten sie es reparier sie reparierten äh machten ein häuschen für die vogel und dann schauten sie draußen wie die vögel äh zwitschern, ja.

Diese Geschichte über einen missglückten Schlittenausflug diente als Überleitung bzw. als Basis für die Erzählung eines eigenen Ferienerlebnisses. Nachstehender Ausschnitt aus einem Transkript ist ein Beispiel für eine solche Ferienerlebniserzählung von einem türkischen Mädchen.
also ich und meine freundinnen gehen in ah fort. und dort dort haben also ins schwimmbad, in hallebad und dort waren wir schwimmen und ich konnte da nicht gut schwimmen und meine schwester auch nicht, und dann und ich habe dann dann habe ich probiert und ich konnte es dann und meine schwester nicht, und dann also meine kollegin ihr bruder war auch mit und er konnte auch nicht schwimmen, dann hat er die schwimmflügel genommen und meine schwester und meine kollegin waren dann ins also waren rein, also für meine schwester war es dann ein bisschen hoch das wasser und dann sind sie reingegangen und dann hat meine kollegin gesagt ich lasse dich jetzt los, dann ist meine schwester, sie konnte nicht schwimmen, dann ist sie herunterge $=$ und sie konnte nicht schwimmen, dann probiert sie immer hoch und sie konnte nicht schwimmen und dann hat N. [anonymisiert], meine kollegin, gerade ihre mutter geruft und dann ist ihre mutter gekommen und hat meine schwester rausgeholt.

Die Interviews dauerten durchschnittlich zwischen acht und zwölf Minuten. Diese gilt es unter besonderer Berücksichtigung der Unterschiede zwischen mündlichem und schriftlichem Sprachgebrauch in Relation $\mathrm{zu}$ den schriftlichen Texten $\mathrm{zu}$ analysieren. Besonderes Augenmerk wird darauf gelegt, ob die Kinder überhaupt in der Lage sind, in der mündlichen Sprachproduktion zwischen Dialekt und Hochsprache zu unterscheiden und darüber hinaus im Stande sind, ein Gespräch konsequent in Hochsprache oder zumindest einer standardnahen Varietät zu führen.

Die Analyse der schriftlichen und mündlichen Daten hinsichtlich morphologischer und syntaktischer Auffälligkeiten soll die folgenden Fragen beantworten: Welche Abweichungen treten am häufigsten auf? Welche davon können auf den Dialekt oder auf die Muttersprache zurückgeführt werden? Wie sieht es hinsichtlich der auftretenden Abweichungen im Vergleich der Sprachgruppen untereinander aus bzw. wie gestaltet sich der Vergleich der fremdsprachigen Kinder mit den Schweizerdeutschen Kindern? 


\subsection{Erste Untersuchungsergebnisse}

Eine Erstauswertung der Aussagen der Kinder über deren Sprachgebrauch und deren Sprachverhalten hat ergeben, dass nicht alle Kinder ihr Sprachverhalten gleich gut reflektieren und die Anwendungsbereiche von Dialekt und Standard angeben können. Meistens unterscheiden die Kinder vorerst nur zwischen ihrer Erstsprache und Deutsch. Im weiteren Verlauf des Gespräches und durch gezieltes Nachfragen können die Kinder durchaus zwischen Mundart und Hochdeutsch unterscheiden.

Diese komplexe Nebeneinander spiegelt sich besonders gut im folgenden Ausschnitt aus einem Interview mit einem albanischen Mädchen wider; das Mädchen hatte soeben erwähnt, dass es drei Geschwister hat, davon zwei älter als es selbst:

I: In welcher Sprache sprichst du mit deinen Geschwistern?

A: manchmal so Deutsch und manchmal Albanisch

I: und mit deinen Eltern?

A: Albanisch

I: und wenn ihr alle zusammen seid zuhause, wie sprecht ihr dann?

A: auch Albanisch

I: und mit den Leuten, die um euch herum wohnen, mit deiner Nachbarschaft, welche Sprachen gebrauchst du mit den Nachbarn?

A: Deutsch, weil fast alle reden deutsch

I: und wenn du jetzt Deutsch sagst. Mhm. Kannst du das noch genauer sagen? ... Sprichst du mit ihnen so wie mit mir hier oder wie mit deinen Freundinnen oder?

A: so Hochdeutsch

I: Hochdeutsch. Und wie sprichst du mit deinen Freundinnen?

A: so normal, so Berndeutsch

I: Und warum sprichst du mit deinen Nachbarn Hochdeutsch?

A: Weil die können nicht= manchmal die Leute die können= die reden nur Hochdeutsch, wenn sie "hallo" oder so sagen.

I: Und mit deinen Freundinnen hier an der Schule?

A: also

I: oder auch mit denen außerhalb von der Schule?

A: Also hier bei uns rede ich mit denen Berndeutsch.

So wie es dieses Mädchen hier schildert, verhält es sich bei vielen Kindern. Im familiären Umfeld wird vorwiegend die Erstsprache gesprochen; teilweise - abhängig vom eigenen Alter und dem der Geschwister - erfolgt die Kommunikation mit Geschwistern auf Deutsch. Da die Kinder mit Freundinnen und Schulkollegen, mit der Nachbarschaft etc. eine Variante des Schweizerdeutschen und in wenigen Fällen Hochdeutsch sprechen, wird durch den zunehmenden Kontakt mit gleichaltrigen Schweizern für das Kind und für seine Geschwister auch der Anteil der Umgebungssprache im Vergleich zur Familiensprache größer. In der Schule sprechen die Kinder vorwiegend (jedoch abhängig von den Gewohnheiten des Lehrpersonals) Hochdeutsch. Angesichts der verschiedenen Situationen, in denen die Varietäten zum Einsatz kommen, ist die Bemerkung zum Sprachgebrauch mit den Freundinnen dennoch nicht verwunderlich. Die Äußerung "so normal - Berndeutsch" lässt darauf schließen, dass das Kind das Standarddeutsche noch als das vom Üblichen Abweichende betrachtet.

Die Schüler haben jedoch mehrheitlich keine Scheu vor der verlangten Verwendung der Hochsprache und beginnen selbstbewusst und ohne Bedenken zu erzählen. Es stellt für sie durchwegs kein Problem dar, das in etwa 12-minütige Gespräch in einer standardnahen Varietät zu führen. Bis auf wenige Ausnahmen - diese vor allem im Bereich des Lexikons wurde wenig zwischen Mundart und Hochsprache geswitcht und es gibt keine grundsätzliche Verwirrung in der Verwendung der Varietäten. 
Die Einstellung gegenüber dem Standarddeutschen scheint sowohl von Seiten der Migrantenkinder als auch von Seiten der Schweizerdeutschen Vergleichsgruppe sehr positiv $\mathrm{zu}$ sein. Es ist hinlänglich bekannt, dass es für Deutschschweizer nicht immer einfach ist, diese positive Einstellung zu bewahren. Häcki Buhofer/Burger (1998: 134) sprechen davon, dass Erwachsene nur durch einen "kommunikativen Notfall" dazu veranlasst werden, Standarddeutsch zu verwenden. Sieber/Sitta (1994: 202) stellen die Entwicklung der Einstellungen anhand dreier Zitate treffend dar:

(1) "Wir müssen Hochdeutsch nicht lernen - wir können es." (Unterstufenschüler)

(2) "Hochdeutsch könnte von mir aus aussterben." (Mittelstufenschüler)

(3) "Gefühle kann ich besser in Mundart ausdrücken. Hochdeutsch ist für mich eine Fremdsprache." (Oberstufenschülerin)

Aufgrund der konsequenten Nutzung der Standardsprache in allen Bereichen des schulischen Alltags kann man hoffen, dass sich die Einstellung der in vorliegender Studie befragten Kinder im Vergleich zu jenen aus bisherigen Untersuchungen festigt.

Um einen ersten Einblick in die schriftliche Produktion zu erhalten, wurden zwei Texte von bislang sechs Kindern, je zwei pro Sprachgruppe, genauer untersucht. Es wurden jeweils schon im Regelunterricht verfasste Texte herangezogen, die eine vergleichbare Textlänge vorweisen.

Die Texte der Kinder mit Migrationshintergrund sind nur in Einzelfällen kürzer als jene der Schweizerdeutschen Vergleichskinder. Schwankungen im Textumfang können somit nicht unmittelbar auf die Tatsache zurückgeführt werden, dass Deutsch als Zweitsprache erworben wird. Die Texte der Migrantenkinder weisen jedoch hinsichtlich der Morphologie (vor allem in den Bereichen Kasusgebrauch, Substantivdeklination, Adjektivdeklination, Bildung des Präteritums, Genus und Numerus) und der Syntax (im Bereich der Satzstellung) mehr Fehler auf.

Bisher wurden in die Fehlerauflistung auch Inkorrektheiten im Bereich der Orthographie erfasst. Die Differenz der Fehleranzahl zwischen deutsch- und fremdsprachigen Kindern ist in diesem Bereich jedoch nicht so groß. D. h. der Bereich der Morphologie und Syntax ist bei den SD-Kindern im Vergleich zu den Nichtmuttersprachlern wahrscheinlich schon weiter entwickelt als jener der Orthographie.Bei Migrantenkindern sind vor allem folgende morphologische und syntaktische Unsicherheiten zu beobachten: Den türkischen Kindern fällt der Gebrauch des Genus schwer. Es ist naheliegend, diese Schwierigkeiten auf das Fehlen des Genus in der Herkunftssprach zurückzuführen sind. Einige Beispiele dazu:

Manchmal, wenn ich die Licht nicht einschalte...

Leider gehört der Zimmer nicht nur mir, sondern...

Sie und sein Mann waren geschieden sie und seine Mutter wohnten zusamen sein Papa aleine der Papa wohnte in so einem Bauernhof. Sie und seine Mutter hatten einen unfal seine Mutter starb aber sie wuste es nicht sein Papa war neben ihr beim Spittahl.

Diese Unsicherheit hinsichtlich des Genus führt auch zu einer deutlich erkennbaren vermehrten Fehleranzahl in den Bereichen Substantiv- und Adjektivdeklination. Vergleichbare Fehler sind bei den Schweizerdeutschen Kindern, aber momentan auch bei jenen aus der Sprachgruppe Bosnisch/Kroatisch/Serbisch seltener.

... ein kleines Tisch hat es und zwei Teppiche.

Am nächsten Tag hatte die Frau ein wenigen Honig und ein Käfer und ein langer Schnur und ein lagngen Seil.

Also nemht er den seil und der Käfer. 
In all diesen Beispielen ist kein expliziter Dialekteinfluss erkenntlich. Die bisher aufgefundenen Abweichungen in den Bereichen der Morphologie und Syntax können nur begrenzt und in Einzelfällen dem Einfluss des lokalen Dialekts zugeschrieben werden (vgl. hierzu die Beispiele im Abschnitt 3). Den ersten vorläufigen Ergebnissen zufolge kann die Frage, ob die diglossische Situation Auswirkungen auf den Zweitspracherwerb hat, nicht klar bejaht werden. In einzelnen Äußerungen sind zwar Dialekteinflüsse erkennbar, allerdings sind diese im Vergleich zu jenen der Erstsprache oder anderer Einflüsse sehr gering.

\section{Zusammenfassung und Ausblick}

Kinder mit Migrationshintergrund ebenso wie Schweizerdeutsche Kinder müssen für schulischen Erfolg differenzierte und normgerechte schriftsprachliche Fähigkeiten entwickeln. Damit Kinder mit komplexen schriftlichen Texten rezeptiv und bestmöglich auch produktiv umgehen lernen, müssen mögliche Hindernisse aufgedeckt und Hilfe zu ihrer Überwindung geboten werden. Da die konkreten Auswirkungen der diglossischen Situation auf den Zweitspracherwerb in bisherigen Untersuchungen weitgehend vernachlässigt wurden, ist es Ziel des Projektes hier eine vorhandene Lücke zu schließen und einen fundierten Beitrag zur Diskussion über den Gebrauch von Dialekt und Standard im schulischen Kontext zu leisten.

Auf zwei unterschiedlichen Alterstufen, nämlich bei Kindergartenkindern und bei Primarschulkindern, wird durch zwei unterschiedliche Herangehensweisen - einer Sprachstandserhebungsmethode und einer Textanalyse - bisher ausstehende Klarheit über den Status des Dialekts und seiner Rolle als möglicher hinderlicher Faktor im Zweitspracherwerb gewonnen. Insgesamt soll das Projekt dazu beitragen, den sprachlichen Förderbedarf von Kindern mit Migrationshintergrund in dialektalem Umfeld zu erheben. Damit soll ein weiterer Schritt auf dem Weg zur bestmöglichen Förderung der spezifisch sprachlichen und allgemeinen schulischen Entwicklung der Kinder gemacht werden.

\section{Literaturangaben}

Ammon, Ulrich et al. (eds.) (2004): Variantenwörterbuch des Deutschen. Berlin.

Apeltauer, Ernst (1997): Grundlagen des Erst- und Fremdspracherwerbs. Eine Einführung. Berlin.

Berthele, Raphael (2004): "Vor lauter Linguisten die Sprache nicht mehr sehen - Diglossie und Ideologie in der deutschsprachigen Schweiz." In: Helen Christen (ed.): Dialekt, Regiolekt und Standardsprache im sozialen und zeitlichen Raum. Wien: 111-136.

Ehlich, Konrad et al. (2005): Anforderungen an Verfahren der regelmäßigen Sprachstandsfeststellung als Grundlage für die frühe und individuelle Förderung von Kindern mit und ohne Migrationshintergrund. Bonn/Berlin: Bundesministerium für Bildung und Forschung (= Bildungsreform, Bd. 11).

Engin, Havva et al. (2004): Kinder lernen Deutsch als zweite Sprache. Berlin.

Ferguson, Charles A. (1959): "Diglossia". Word 15: 325-340.

Fried, Lilian (2004): Expertise zu Sprachstandserhebungen für Kindergartenkinder und Schulanfänger. Eine kritische Betrachtung. München: Deutsches Jugendinstitut.

Graf, Peter (1987): Frühe Zweisprachigkeit und Schule. Empirische Grundlagen zur Erziehung von Minderheitenkindern. München.

Gyger, Mathilde (2005): Projekt Standardsprache im Kindergarten (PSS). Schlussbericht. Basel: Rektorat Kindergärten Basel, Arbeitsgruppe Integration.

Häcki Buhofer/Burger, Harald (1998): Wie Deutschschweizer Kinder Hochdeutsch lernen. Der ungesteuerte Erwerb des gesprochenen Hochdeutschen durch Deutschschweizer Kinder zwischen sechs und acht Jahren. Stuttgart. (= Zeitschrift für Dialektologie und Linguistik: Beihefte; Heft 98). 
Das Projekt "Deutsch als Zweitsprache in Dialektumgebung"

Knapp, Werner (1997): Schriftliches Erzählen in der Zweitsprache. Tübingen: Niemeyer.

Kolde, Gottfried (1981): Sprachkontakte in gemischtsprachigen Städten. Vergleichende Untersuchungen über Voraussetzungen und Formen sprachlicher Interaktion verschiedensprachiger Jugendlicher in den Schweizer Städten Biel/Bienne und Fribourg/Freiburg i. Ue. Wiesbaden. (= Zeitschrift für Dialektologie und Linguistik: Beihefte; Heft 37).

Landert, Karin (2006): "Standarddeutsch im Vorschulalter. Ein Schweizerdeutsch- und ein Standarddeutsch-Kindergarten im Vergleich. " In: Dürscheid, Christa/Businger, Martin (eds.): Schweizer Standarddeutsch. Beiträge zur Varietätenlinguistik. Tübingen: 211-231.

Mitchell, Rosamond/Myles, Florence (2004): Second language learning theories. Second edition. London.

Nodari, Claudio/De Rosa, Raffaele (2003): Mehrsprachige Kinder. Ein Ratgeber für Eltern und andere Bezugspersonen. Bern.

Penner, Zvi (2005): Auf dem Weg zur Sprachkompetenz. Neue Perspektiven der sprachlichen Frühförderung bei Migrantenkindern. Frauenfeld.

PHZH und EDK-Ost (eds.) (2005): Hinweise zur Beobachtung von Kindern mit Deutsch als Zweitsprache (DaZ) in der Grund- und Basisstufe resp. im Kindergarten und in der 1./2. Klasse. http://www.ag.ch/gbs/shared/dokumente/pdf/5_-_hinweise_zu_deutsch_als_zweit sprache.pdf, Stand 20.5.2005.

Pulver, Bernhard (2007): "Fertig Bärndütsch?" e-ducation, Amtliches Schulblatt des Kantons Bern 1/2007: 14-15.

Reich, Hans H. et al. (2002): Spracherwerb zweisprachig aufwachsender Kinder und Jugendlicher. Ein Überblick über den Stand der nationalen und internationalen Forschung. Hamburg: Behörde für Bildung und Sport.

Rosenberg, Peter (1986): Der Berliner Dialekt und seine Folgen für die Schüler. Geschichte und Gegenwart der Stadtsprache Berlins sowie eine empirische Untersuchung zu den Schulproblemen dialektsprechender Schüler. (Diss.) Tübingen.

Schmidlin, Regula (1999): Wie Deutschschweizer Kinder schreiben und erzählen lernen. Textstruktur und Lexik von Kindertexten aus der Deutschschweiz und aus Deutschland. Tübingen/Basel.

Sieber, Peter/Sitta, Horst (1994): "Zur Rolle der Schule beim Aufbau von Einstellungen zu Dialekt und Standardsprache." In: Burger, Harald/Häcki Buhofer, Annelies (eds.): Spracherwerb im Spannungsfeld von Dialekt und Hochsprache. Bern etc.: 199-213.

Stöckling, Hans Ulrich (2005): "Mundart in der Volksschule." Forum Helveticum 15: 82-87.

Wegener, Heide (1994): "Der Einfluss des Augsburger Dialekts auf den Erwerb der Morphosyntax des Deutschen durch Grundschulkinder aus Polen, Russland und der Türkei." In: Burger, Harald/Häcki Buhofer, Annelies (eds.): Spracherwerb im Spannungsfeld von Dialekt und Hochsprache. Bern etc.: 89-106.

Werlen, Iwar (1998): "Mediale Diglossie oder asymmetrische Zweisprachigkeit? Mundart und Hochsprache in der deutschen Schweiz." Babylonia 1: 22-35.

\section{Sprachstandsmessungsverfahren}

Bärenstark (2002) = Senatsverwaltung für Schule Jugend und Sport (ed.) (2002): Bärenstark. Berliner Sprachstandserhebung und Materialien zur Sprachförderung für Kinder in der Schuleingangsphase. Berlin.

CITO (2007) $=$ Cito Deutschland GmbH (ed.) (2007): Cito-Sprachtest. Butzbach.

Deutsch Plus (2006) = Senatsverwaltung für Bildung, Jugend und Sport (ed.) (2006): Den Sprachstand vor Schuleintritt erfassen. Erhebungsinstrument 2006. Deutsch Plus. Berlin.

Fit in Deutsch (2006) = Niedersächsisches Kultusministerium (ed.) (2006): Fit in Deutsch. Feststellung des Sprachstandes. Hannover. 
HAVAS 5 (2003) = Reich, Hans/Roth, Hans-Jochen (2003): HAVAS 5. Hamburger Verfahren zur Analyse des Sprachstandes Fünfjähriger. Auswertungsbogen und Auswertungshinweise. Hamburg: Landesinstitut für Lehrerbildung und Schulentwicklung.

Kenntnisse in Deutsch als Zweitsprache (2002) = Staatsinstitut für Schulpädagogik und Bildungsforschung (ed.) (2002): Kenntnisse in Deutsch als Zweitsprache erfassen. Screening-Modell für Schulanfänger. München.

MSS (2003) = Holler-Zittlau, Inge/Dux, Winfried/Berger, Roswitha (2003): Marburger Sprach-Screening für 4- bis 6-jährige Kinder (MSS). Ein Sprachprüfverfahren für Kindergarten und Schule. Horneburg.

SFD (2006) = Hobusch, Anna/Lutz, Nevin/Wiest, Uwe (2006): Sprachstandsüberprüfung und Förderdiagnostik für Ausländer- und Aussiedlerkinder. (SFD). Horneburg.

SISMIK (2004) = Ulich, Michaela/Mayr, Toni (2004): Sismik. Sprachverhalten und Interesse an Sprache bei Migrantenkindern in Kindertageseinrichtungen. Begleitheft zum Beobachtungsbogen sismik. Freiburg im Breisgau.

SSV (2003) = Grimm, Hannelore (2003): Sprachscreening für das Vorschulalter. Kurzform des SETK 3-5. Göttingen. 